\title{
The Molecular Mechanism of the Catalase-like Activity in Horseradish Peroxidase
}

\author{
Pablo Campomanes, ${ }^{\dagger, \|}$ Ursula Rothlisberger, ${ }^{\dagger}$ Mercedes Alfonso-Prieto, ${ }^{*, \dagger}$ and Carme Rovira ${ }^{*,+, \S}$
}

${ }^{\dagger}$ Laboratory of Computational Chemistry and Biochemistry, Institute of Chemical Sciences and Engineering, École Polytechnique Fédérale de Lausanne (EPFL), CH-1015 Lausanne, Switzerland

${ }^{\ddagger}$ Departament de Química Orgànica \& Institut de Química Teòrica i Computacional (IQTCUB), Universitat de Barcelona, Martí i Franquès 1, 08208 Barcelona, Spain

${ }^{\S}$ Institució Catalana de Recerca i Estudis Avançats (ICREA), Passeig Lluís Companys, 23, 08018 Barcelona, Spain

Supporting Information
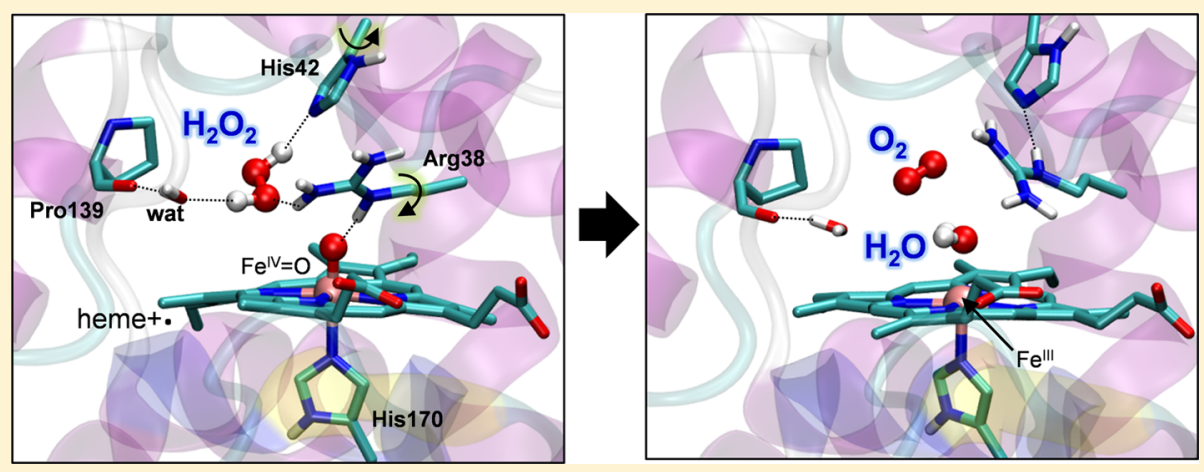

ABSTRACT: Horseradish peroxidase (HRP) is one of the most relevant peroxidase enzymes, used extensively in immunochemistry and biocatalysis applications. Unlike the closely related catalase enzymes, it exhibits a low activity to disproportionate hydrogen peroxide $\left(\mathrm{H}_{2} \mathrm{O}_{2}\right)$. The origin of this disparity remains unknown due to the lack of atomistic information on the catalase-like reaction in HRP. Using QM(DFT)/MM metadynamics simulations, we uncover the mechanism for reduction of the HRP Compound I intermediate by $\mathrm{H}_{2} \mathrm{O}_{2}$ at atomic detail. The reaction begins with a hydrogen atom transfer, forming a peroxyl radical and a Compound II-like species. Reorientation of the peroxyl radical in the active site, concomitant with the transfer of the second hydrogen atom, is the rate-limiting step, with a computed free energy barrier (18.7 $\mathrm{kcal} / \mathrm{mol}, \sim 6 \mathrm{kcal} / \mathrm{mol}$ higher than the one obtained for catalase) in good agreement with experiments. Our simulations reveal the crucial role played by the distal pocket residues in accommodating $\mathrm{H}_{2} \mathrm{O}_{2}$, enabling formation of a Compound II-like intermediate, similar to catalases. However, out of the two pathways for Compound II reduction found in catalases, only one is operative in HRP. Moreover, the hydrogen bond network in the distal side of HRP compensates less efficiently than in catalases for the energetic cost required to reorient the peroxyl radical at the rate-determining step. The distal Arg and a water molecule in the "wet" active site of HRP have a substantial impact on the reaction barrier, compared to the "dry" active site in catalase. Therefore, the lower catalase-like efficiency of heme peroxidases compared to catalases can be directly attributed to the different distal pocket architecture, providing hints to engineer peroxidases with a higher rate of $\mathrm{H}_{2} \mathrm{O}_{2}$ disproportionation.

\section{INTRODUCTION}

Catalases and peroxidases are oxidoreductase enzymes that can be found in nearly all aerobically respiring organisms. They play a crucial protective role in preventing the oxidative damage of cellular components caused by hydrogen peroxide $\left(\mathrm{H}_{2} \mathrm{O}_{2}\right)$, a byproduct from the essential aerobic metabolism, as well as its highly reactive decomposition products. Both enzymes are able to catalyze the heterolytic cleavage of the peroxidic bond, therefore avoiding the accumulation of reactive oxygen species (ROS) in cells and also regulating their concentration in cellular signaling pathways. ${ }^{1}$ In addition, peroxidases, in particular horseradish peroxidase (HRP), employ $\mathrm{H}_{2} \mathrm{O}_{2}$ to oxidize organic substrates. Hence, HRP is extensively used in the fields of immunochemistry, biocatalysis, bioremediation and medicine. ${ }^{2}$

The enzymatic cycle of both heme catalases and peroxidases (Figure 1) is initiated with the $\mathrm{H}_{2} \mathrm{O}_{2}$-mediated oxidation of the resting state of the enzyme (Por-Fe ${ }^{\mathrm{III}}$, where Por stands for the heme porphyrin) to form a high-valent iron-oxo intermediate known as Compound I ( $\mathrm{Cpd} \mathrm{I}) .{ }^{3} \mathrm{Cpd} \mathrm{I}$ is generally described as an oxoferryl porphyrin radical cation $\left(\mathrm{Por}^{\bullet+}-\mathrm{Fe}^{\mathrm{IV}}=\mathrm{O}\right){ }^{4}$ However, the reactivity of this intermediate differs between heme catalases and peroxidases. Peroxidases preferentially

Received: June 30, 2015

Published: August 14, 2015 


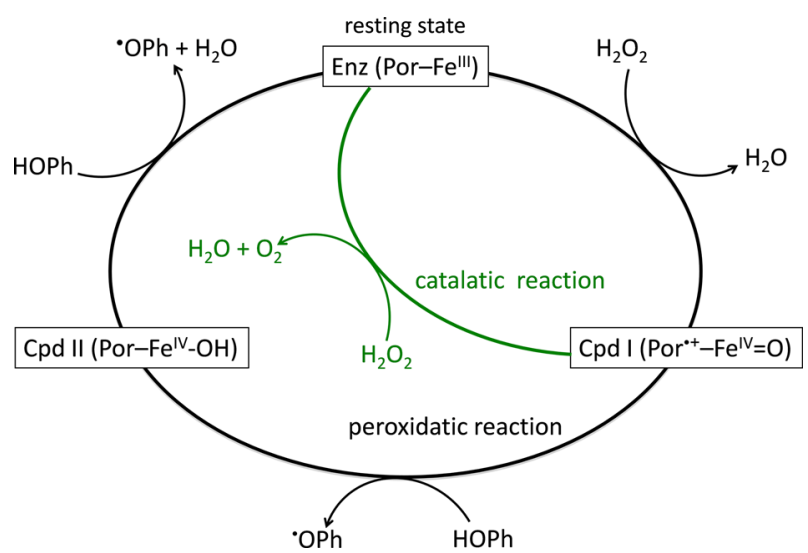

Figure 1. Reactions catalyzed by peroxidases and catalases using hydrogen peroxide $\left(\mathrm{H}_{2} \mathrm{O}_{2}\right)$. The catalytic cycle begins with Compound I (Cpd I) formation for both oxidoreductase enzymes. In catalases, Cpd I is reduced back to the resting state by a second molecule of $\mathrm{H}_{2} \mathrm{O}_{2}$ (catalatic reaction). In contrast, peroxidases preferentially recover the resting state via the peroxidatic reaction, in which Cpd I sequentially reacts with two molecules of a one-electron donating substrate (e.g., phenol, $\mathrm{PhOH}$ ), passing through another intermediate named Compound II (Cpd II).

catalyze Cpd I reduction by one-electron donating substrates, typically phenols or aromatic amines, to recover the native ferric state either directly or via an intermediate named Compound II (Cpd II). ${ }^{5} \mathrm{Cpd}$ II is usually described as an oxo/hydroxoferryl heme intermediate (Por- $\mathrm{Fe}^{\mathrm{IV}}=\mathrm{O} / \mathrm{Por}-\mathrm{Fe}^{\mathrm{IV}}-\mathrm{OH}$ ). In contrast, monofunctional heme catalases use a second $\mathrm{H}_{2} \mathrm{O}_{2}$ molecule to rapidly reduce $\mathrm{Cpd} \mathrm{I}$ to the resting state, releasing $\mathrm{O}_{2}{ }^{6}$. Therefore, both enzymes are able to degrade hydrogen peroxide, even though their preferred mechanistic route varies (Figure 1). Catalases catalyze $\mathrm{H}_{2} \mathrm{O}_{2}$ dismutation (catalatic reaction), whereas peroxidases use $\mathrm{H}_{2} \mathrm{O}_{2}$ for substrate oxidation (peroxidatic reaction). Nevertheless, catalase-like activity, i.e., release of $\mathrm{O}_{2}$ in the presence of $\mathrm{H}_{2} \mathrm{O}_{2}$, has been observed for some peroxidases, e.g., horseradish peroxidase (HRP), in the absence of one-electron donors. Why $\mathrm{H}_{2} \mathrm{O}_{2}$ dismutation is an extremely efficient process in catalases, but takes place at a much slower pace in peroxidases, ${ }^{5 b, 7}$ remains unknown.

Knowledge of the factors that determine the differences between the reactivity of $\mathrm{Cpd} \mathrm{I}$ in heme catalases and peroxidases can be very valuable to assist the rational design of novel biocatalysts and biomimetic compounds able to mediate a large range of oxidative transformations. ${ }^{8}$ It is thus crucial to understand the mode of action of these enzymes to unambiguously characterize the molecular features that lie at the origin of this distinct Cpd I reactivity. ${ }^{5 b, 9}$ In general, two aspects have been suggested ${ }^{10}$ to play a major role in Cpd I reactivity in hemeproteins: (i) solvent accessibility of the active site and (ii) structural and sequence differences in the heme active sites (Figure 2). While peroxidases exhibit an open and solvent-exposed active site, the heme of catalases is deeply buried in the enzyme and the access channel is long and narrow. Besides, both oxidoreductase families have different active site residues. ${ }^{11}$ In catalases, the proximal axial ligand is Tyr, whereas it is His in peroxidases. Furthermore, the main distal residues His/Asn in catalases are replaced by His/Arg in peroxidases (Figure 2). In addition, the distal His side chain is oriented nearly parallel to the heme group in catalases, whereas in peroxidases it is positioned approximately orthogonal to the plane defined by the heme. (a)

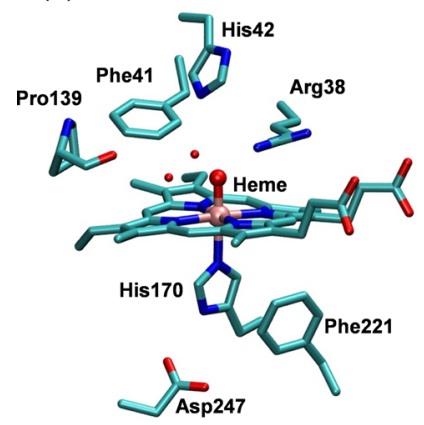

(b)

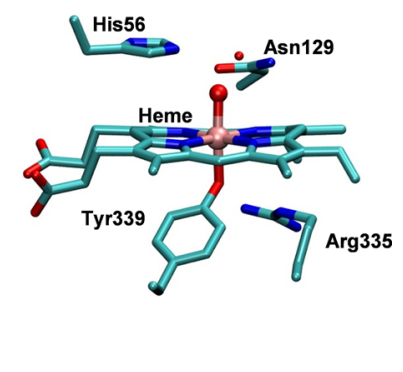

Figure 2. Active sites of (a) horseradish peroxidase and (b) a monofunctional catalase, based on the crystal structures of HRP Cpd $\mathrm{I}(\mathrm{PDB} \text { entry } 1 \mathrm{HCH})^{17}$ and Helicobacter pylori catalase Cpd I (PDB entry 2IQF). ${ }^{46}$

Recent experimental ${ }^{12}$ and theoretical ${ }^{13}$ studies have shown that the distinct reactivity of $\mathrm{Cpd} \mathrm{I}$ toward $\mathrm{H}_{2} \mathrm{O}_{2}$ in these enzymes cannot be explained in terms of differences in the nature of the proximal ligands, therefore pointing to the characteristic properties of the distal residues as the main cause for this diverse behavior.

The mechanism of $\mathrm{Cpd} \mathrm{I}$ reduction by $\mathrm{H}_{2} \mathrm{O}_{2}$ in two monofunctional heme catalases, Helicobacter pylori catalase (HPC) and Penicillium vitale catalase (PVC) was previously investigated using quantum mechanical/molecular mechanical (QM/MM) molecular dynamics (MD) simulations. ${ }^{14}$ It was shown that the reaction does not proceed by a proton and hydride transfer, as has often been assumed, but rather by two sequential one electron transfers. In a first step (Figure 3), $\mathrm{H}_{2} \mathrm{O}_{2}$ transfers one hydrogen atom to the oxoferryl of $\mathrm{Cpd} \mathrm{I}$, forming a Cpd II-like species (Por-Fe ${ }^{\mathrm{IV}}-\mathrm{OH}$ ). To complete the reaction, two pathways (named $\mathbf{A}$ and $\mathbf{B}$ ) with very similar free energy barriers $\left(\sim 12 \mathrm{kcal} \mathrm{mol}^{-1}\right)$ can be operative. Pathway A involves the distal His as an acid-base catalyst, as proposed by Fita and Rossmann, ${ }^{15}$ shuttling a proton from the peroxyl radical to the hydroxoferryl, concomitantly with an electron transfer (Hismediated pathway or A). A variation of this mechanism, involving an ionizable tryptophan distal residue, was recently proposed for bifunctional catalase-peroxidases, ${ }^{16}$ which also disproportionate $\mathrm{H}_{2} \mathrm{O}_{2}$ at a high pace. Alternatively, the hydrogen atom can transfer directly to the hydroxoferryl by rotation of the peroxyl radical in the active site (direct pathway or $\mathbf{B}$ ).

The lack of a similar kind of detailed atomistic level information in monofunctional peroxidases precludes understanding the factors underlying their low catalase-like activity. Here we present a $\mathrm{QM}(\mathrm{DFT}) / \mathrm{MM} \mathrm{MD}$ investigation of the mechanism of Cpd I reduction by $\mathrm{H}_{2} \mathrm{O}_{2}$ in $\mathrm{HRP}$, one of the most relevant and historically most important heme peroxidases. HRP is also the plant peroxidase with the highest overall turnover of $\mathrm{H}_{2} \mathrm{O}_{2} \cdot{ }^{7 b}$ We show that Cpd I reduction in HRP can follow only one out of the two pathways operative in catalase, the direct pathway, in which the two hydrogen atoms of $\mathrm{H}_{2} \mathrm{O}_{2}$ are directly and sequentially transferred to the oxoferryl oxygen, without participation of the distal His. Despite the similar mechanism, the energy barrier of the reaction is higher in HRP than in catalase due to the particular network of hydrogen bond interactions among the peroxide and the active site residues. This explains why HRP, and peroxidases in general, are less efficient than catalases to dismutate hydrogen peroxide. Analysis of the reaction pathway provides insight into the question how 


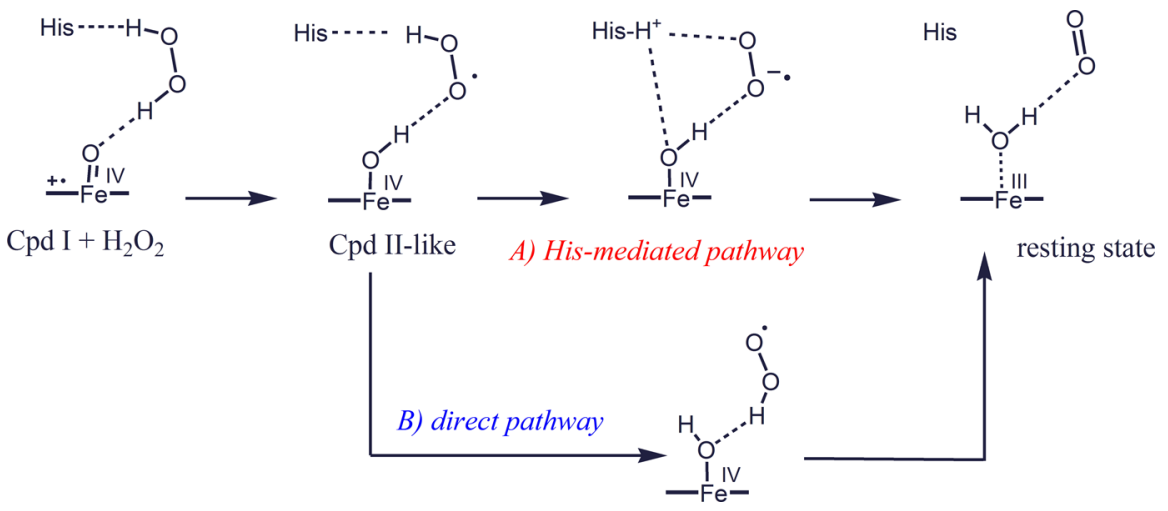

Figure 3. Mechanism of Compound I reduction by $\mathrm{H}_{2} \mathrm{O}_{2}$ in monofunctional catalases. ${ }^{14}$ Two possible pathways were found using metadynamics simulations.

peroxidases have evolved to minimize the catalatic reaction and use other reducing substrates instead.

\section{METHODS}

2.1. Classical Molecular Dynamics. The model used as starting configuration for the classical MD simulations was built based on the Xray structure of HRP Cpd I (PDB code: $1 \mathrm{HCH}) .{ }^{17}$ The crystal structure was immersed in a periodic box of approximately $80 \times 86 \times 72 \AA^{3}$ containing about 14000 water molecules, and neutralized with $1 \mathrm{Na}^{+}$ counterion. The box dimensions were chosen to achieve a minimum distance of $24 \AA$ between periodically replicated images of the protein. Conventional $(\mathrm{pH}=7)$ protonation states were assigned to all titratable protein residues using propKa, ${ }^{18}$ and further verified through inspection of their hydrogen bond pattern and nearby chemical environment. Accordingly, His42 in the distal active site pocket of the enzyme was chosen as $\mathrm{N}_{\delta}$-protonated, which is the commonly assumed protonation state for this residue in horseradish peroxidase at neutral $\mathrm{pH}^{19}$ This choice is also in agreement with the fact that the catalase-like activity of HRP occurs at $\mathrm{pH}>6.4 .^{7 \mathrm{~b}}$ In fact, the drop of catalase activity at $\mathrm{pH}<$ 6.4 has been attributed to double-protonation of His $42 .{ }^{7 \mathrm{~b}}$ Consistently, the distal His has been found to be doubly protonated (i.e., positively charged) for cytochrome $\mathrm{c}$ peroxidase at slightly acidic conditions $(\mathrm{pH}$ $6.0)^{20}$

The parm99SB parametrization of the all-atom AMBER force field ${ }^{21}$ was used to model standard protein residues and counterions, whereas the TIP3P model $^{22}$ was employed for water molecules. For the heme $b$ prosthetic group in Cpd I, the bonded parameters by $\mathrm{Banci}^{23}$ were adopted, whereas the RESP charges were derived from quantum chemical computations performed at the density functional theory level (B3LYP/6-31G*) on a model system consisting of the prosthetic group and some of the protein residues next to it (the proximal residues His170 and Asp247, as well as its H-bonding network formed by Gln176, Arg31 and Ser35). Electrostatic interactions were taken into account using the Particle Mesh Ewald (PME) algorithm ${ }^{24}$ with a real space cutoff of $10 \AA$. The same cutoff was employed for the treatment of the van der Waals interactions. An integration time step of $1 \mathrm{fs}$ was used. The physiological temperature of $310 \mathrm{~K}$ was achieved by coupling the system to a Langevin thermostat. ${ }^{25}$ After minimization and careful thermalization of the HRP Cpd I structure in water solution, an initial classical MD simulation was run for $5 \mathrm{~ns}$. It was found that water molecules entered and left the active site cavity several times during this simulation via the channel that extends from Pro139 to the bulk solvent. This is in agreement with the conclusions of previous experimental ${ }^{17,26}$ and computational studies ${ }^{27}$ on HRP, which have addressed the problem of the accessibility of solvent molecules to the distal pocket and their role in Cpd I formation.

A hydrogen peroxide molecule was accommodated into the active site pocket between Pro139 and His42, H-bonded to this latter residue, through replacement of one of the water molecules present in the cavity (configuration 0 in Figure 4). A 10 ns MD simulation in the canonical (NVT) ensemble was performed to relax the $\mathrm{Cpd} \mathrm{I}-\mathrm{H}_{2} \mathrm{O}_{2}$ complex. A
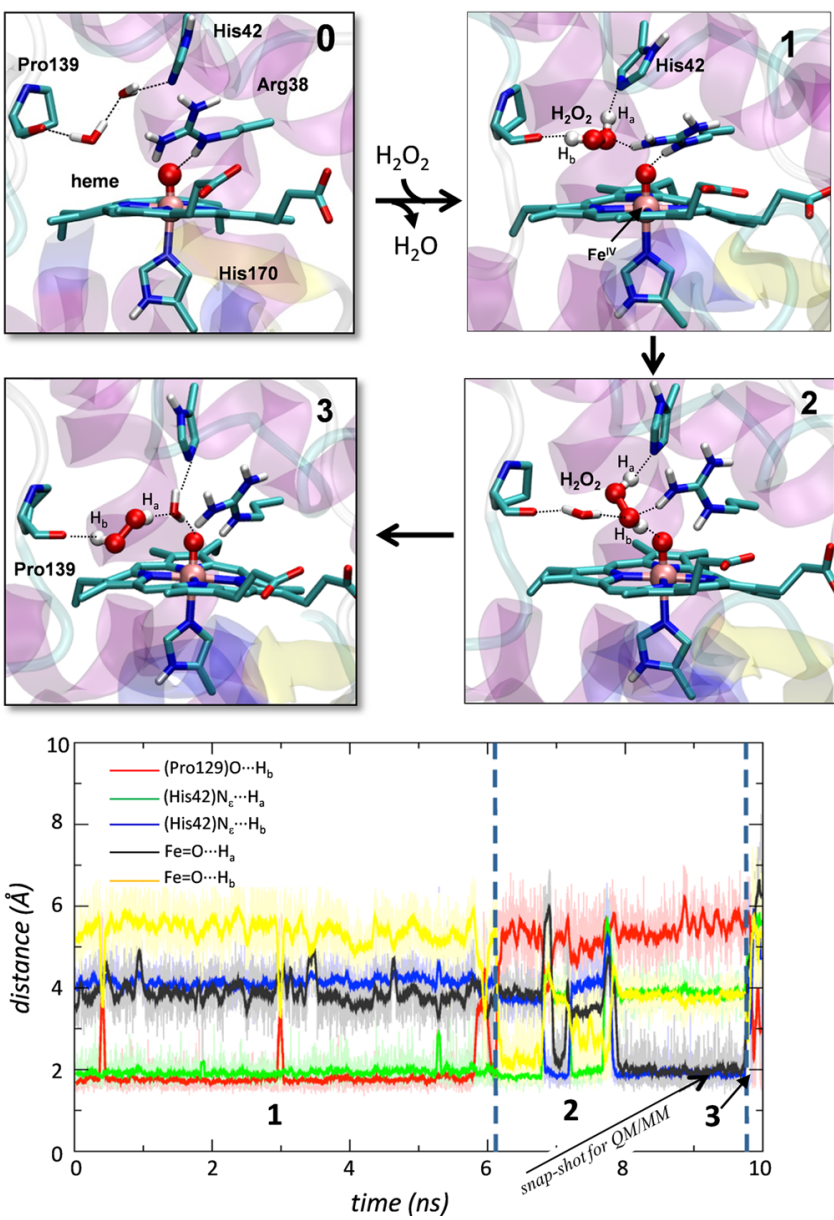

Figure 4. Classical molecular dynamics (MD) simulations of the HRP Compound I: hydrogen peroxide complex. The three configurations explored by $\mathrm{H}_{2} \mathrm{O}_{2}$ in the active site (labeled 1-3) are displayed on top, while the time evolution of the relevant distances describing the interactions of $\mathrm{H}_{2} \mathrm{O}_{2}$ with the active site residues is shown at the bottom. Configuration 0 (top left panel) is a snapshot of the simulation prior to introducing a molecule of hydrogen peroxide in the active site.

configuration was extracted from this simulation to initiate the subsequent $\mathrm{QM} / \mathrm{MM} \mathrm{MD}$ study. All the classical MD simulations were carried out using the NAMD package. ${ }^{28} \mathrm{VMD}^{29}$ was used for visualization, analysis and image generation.

2.2. QM/MM Molecular Dynamics. The QM/MM implementation employed in this study combines the use of the QM program QUICKSTEP $^{30}$ and the MM driver FIST, both forming part of the 
CP2K package (freely available at www.cp2k.org, released under GPL license). In this code, the general QM/MM scheme is based on a real space multigrid technique to compute the electrostatic coupling between the $\mathrm{QM}$ and $\mathrm{MM}$ regions. ${ }^{31} \mathrm{~A}$ quantum region (consisting of the oxoferryl porphyrin radical cation, the $\mathrm{H}_{2} \mathrm{O}_{2}$ molecule, the distal $\mathrm{Arg} 38$ and His42 residues, the proximal His 170, and an additional water molecule that is hydrogen bonded to $\mathrm{H}_{2} \mathrm{O}_{2}$ ) was treated at the DFT level, whereas the remaining part of the system, including water molecules and counterions, was modeled at the classical level using the AMBER force field. Further support for the requirement of including all these residues as part of the QM subsystem can be found in a previous QM/MM study on HRP Cpd I. ${ }^{3 a}$ The valence of the terminal QM atoms was saturated by the addition of capping hydrogen atoms. A dual basis set, Gaussian and plane-wave (GPW) formalism, was employed to compute the interaction energy within the atoms belonging to the QM region. In particular, a double- $\zeta$ valence basis set augmented with a set of polarization functions (DZVP) $)^{32}$ was used in order to get an accurate description of the wave function of the QM subsystem, while an auxiliary plane-wave basis set expanded up to a density cutoff of $320 \mathrm{Ry}$ was utilized to converge the electron density in conjunction with GoedeckerTeter-Hutter (GTH) pseudopotentials ${ }^{33}$ to describe the core electrons. Exchange and correlation energies were computed within the GGA approximation using the BLYP functional. ${ }^{34}$

The self-interaction error (SIE), inherent to this methodology, may result in a poor description of open-shell species. Hence, we performed single-point calculations on representative structures along the reaction pathway using the hybrid B3LYP functional, which is known to be less affected by the SIE. The obtained spin densities (Table 1 and Figure S2) are very similar to those obtained with BLYP, indicating that this particular case does not seem to be affected significantly by the known limitations of GGA DFT. A similar conclusion was reached in our previous work on catalase. ${ }^{14}$ A quartet spin state was considered to carry out all the QM/MM MD simulations. This decision was motivated from previous computational ${ }^{14}$ and experimental studies, ${ }^{4 b, 35}$ which found the quartet state to be the relevant one for the catalatic reaction in catalases (since the crossing from the quartet to the higher spin states characteristic of the enzyme resting state took place at the products state and not during the reaction) ${ }^{14}$ The quartet and doublet states are nearly degenerated in HRP Cpd I; ${ }^{\mathrm{Sa}, 19}$ therefore, it is reasonable to assume that the catalase-like activity of HRP takes place on the quartet spin state surface as well.

Table 1. Distances and Number of Unpaired Electrons $\left(\boldsymbol{n}^{\text {spin }}\right)^{a}$ at the Local Minima of the Free Energy Surface of Figure 5

\begin{tabular}{lllll} 
& \multicolumn{1}{c}{ reactants } & \multicolumn{1}{c}{ A2 } & \multicolumn{1}{c}{$\mathrm{B} 3$} & \multicolumn{1}{c}{ products } \\
$\mathrm{Fe}-\mathrm{O}(\AA)$ & $1.68 \pm 0.19$ & $1.70 \pm 0.26$ & $1.83 \pm 0.27$ & $3.10 \pm 0.76$ \\
$\mathrm{O}-\mathrm{O}(\AA)$ & $1.50 \pm 0.17$ & $1.37 \pm 0.21$ & $1.38 \pm 0.20$ & $1.25 \pm 0.17$ \\
$\mathrm{FeO}\left(n^{\text {spin }}\right)$ & $2.1-2.1$ & $2.0-2.1$ & $1.7-2.0$ & $0.9-1.0$ \\
$\mathrm{OO}\left(n^{\text {spin }}\right)$ & $0.0-0.2$ & $0.8-0.9$ & $0.9-1.0$ & $1.9-2.0$ \\
Porph $\left(n^{\text {spin }}\right)$ & $0.7-1.0$ & $0.0-0.2$ & $0.0-0.3$ & $0.0-0.1$
\end{tabular}

${ }^{a}$ The spin density was integrated using Bader's atoms in molecules theory. The variation between BLYP and B3LYP functionals is given.

All the ab initio MD simulations were performed with BornOppenheimer dynamics in the NVT ensemble and using an integration time step of $0.5 \mathrm{fs}$. The $\mathrm{QM}$ region was initially relaxed by performing a geometry optimization while the MM part was kept frozen. Then the system was thermalized and further equilibrated without any constraint during about 2 ps employing a stochastic velocity rescaling thermostat ${ }^{36}$ to maintain the temperature at $310 \mathrm{~K}$. A representative snapshot was subsequently selected to initiate the metadynamics simulation.

2.3. Metadynamics. The direct formulation of the metadynamics algorithm ${ }^{37}$ was used to enhance the sampling of the phase space and estimate the free energy landscape associated with the reaction under study. This algorithm relies on the addition of a repulsive Gaussian-like potential to the regions of space previously visited, discouraging the system from re-exploring the conformations already sampled during the dynamics and, thereby, accelerating the exploration of other regions. Two collective variables (CV1 and CV2) were chosen to reconstruct the free energy landscape: (i) the coordination number between the two oxygen atoms and the two hydrogen atoms of the $\mathrm{H}_{2} \mathrm{O}_{2}$ molecule, which gives an indication of the degree of $\mathrm{O}_{2}$ formation (one of the final products of the reaction), and (ii) the coordination number between the oxoferryl oxygen atom and the hydrogen atoms of both the $\mathrm{H}_{2} \mathrm{O}_{2}$ and one $\mathrm{H}_{2} \mathrm{O}$ molecule located in the active site cavity (labeled wat), which measures the degree of formation of the new water molecule (the other product of the reaction under study). Similar CVs were previously employed to investigate the same process in heme catalases. ${ }^{14}$ The following definition for the coordination number $(\mathrm{CN})$ of a given species $A$ with respect to another species $B$ was adopted:

$$
C N_{A B}=\sum_{i=1}^{N_{A}} \sum_{j=1}^{N_{B}} \frac{1-\left(r_{i j} / d_{A B}\right)^{p}}{1-\left(r_{i j} / d_{A B}\right)^{q}}
$$

where $N_{A}$ and $N_{B}$ are the number of atomic species $A$ and $B$, respectively, $r_{i j}$ are interatomic distances, and $p, q, d_{A B}$ are the parameters that characterize the decay of the function. In our simulations, we used $A=$ $\left[\mathrm{O}_{a}, \mathrm{O}_{\mathrm{b}}\right], B=\left[\mathrm{H}_{a}, \mathrm{H}_{\mathrm{b}}\right], d_{A B}=1.0 \AA, p=12, q=15$ to describe CV1, whereas the parameters employed in CV2 definition were $A=[\mathrm{O}], B=$ $\left[\mathrm{H}_{\mathrm{a}}, \mathrm{H}_{\mathrm{b}}, \mathrm{H}_{\text {wat }}\right], d_{A B}=1.8 \AA, p=3, q=6$. The width of the Gaussianshaped potential hills was set to 0.05 and 0.08 for CV1 and CV2, respectively, while their height was set to $0.6 \mathrm{kcal} \mathrm{mol}^{-1}$. The time deposition interval between two consecutive Gaussians was set to 15 fs. A total of 400 Gaussian hills were deposited. The simulation was stopped once the products well was sampled and the oxygen molecule was escaping from the active site, which precluded the system to diffuse back to the reactants. Given that only one walker was used to estimate the FEL and the values of the metadynamics parameters chosen, the error in the energy barrier is expected to be $\leq 1.9 \mathrm{kcal} / \mathrm{mol} .^{38}$ This is of the same order as the error of the FEL in catalase,${ }^{14}$ for which similar metadynamics parameters were used, but recrossing, which did not affect the reaction mechanism, was observed.

2.4. Calculation of Active Site Hydrogen Bond Interaction Energies. Additional gas-phase calculations on active site model systems were performed to identify the factors that cause the distinct reactivity of Cpd I toward $\mathrm{H}_{2} \mathrm{O}_{2}$ in $\mathrm{HRP}$ and catalase. To this end, we built simplified active site cluster models corresponding to the minimum and transition state configurations of the rate-limiting step of the reaction. In particular, the models comprised the heme (modeled as iron-porphyrin), the proximal residue (Tyr339 in catalase and His170 in HRP, modeled as methylphenolate and methylimidazole, respectively), the water molecules in the active site cavity (only present in HRP), the $\mathrm{H}_{2} \mathrm{O}_{2}$ substrate (present as a peroxyl radical in the rate-limiting step), and the distal residues capable of interacting with these molecules (Asn129 and His56 in catalase and Arg38 and Pro139 in HRP, modeled as acetamide, methylimidazole, methylguanidinium and 2-acetylpyrrolidine, respectively). Only the side chains up to the $\mathrm{C}_{\beta}$ atom of the active site residues were included, and their valences were saturated using hydrogen atoms. The rest of the protein environment was not considered.

The fragments defined, together with the main hydrogen bond interactions connecting them at the configurations examined, are depicted in Figure S1. The arginine residue in HRP was considered in the same fragment as the heme, as there is a strong salt bridge between the two groups. For each of the fragments in Figure S1, the fragment was displaced $7 \AA$ away (a distance in which the interaction between fragments can be considered as negligible) from the peroxyl radical or the water molecule, while keeping the rest of the fragments at their original position; this procedure was repeated for all fragments, one at a time (the ordering in which the fragments are displaced does not affect the calculated interaction energies). Moreover, the spin density distribution of the system remains the same (i.e., one unpaired electron on the peroxyl radical and two unpaired electrons on the hydroxoferryl group of Cpd II), and thus there are no electronic changes that could affect the calculated interaction energies. The interaction energy $(\Delta E)$ was calculated as the energy difference between the gas phase cluster model with all fragments at their original positions and the same model 
when the distance between the fragment and the peroxyl radical or water molecule is about $7 \AA$. Similar decomposition approaches have been successfully used by others ${ }^{39}$ to rationalize reactivity trends in terms of the deformation and interaction energy changes undergone by the reactants.

\section{RESULTS AND DISCUSSION}

3.1. Dynamics of $\mathrm{H}_{2} \mathrm{O}_{2}$ in the Active Site of HRP. As a first step in our study, we investigated the dynamics of hydrogen peroxide in the active site of HRP by classical MD. Three different modes of interaction between the $\mathrm{H}_{2} \mathrm{O}_{2}$ molecule and the active site residues were observed (Figure 4). Initially, the peroxide molecule is hydrogen bonded to Pro139 and His42 (configuration 1, Figure 4). At $\sim 6 \mathrm{~ns}$, the $\mathrm{H}_{2} \mathrm{O}_{2}$ molecule undergoes a small rotation (configuration 2), breaking the hydrogen bonding interaction with Pro139 and forming two new hydrogen bonds, one with the oxygen atom of the oxoferryl group, and another one with a water molecule (labeled wat) that enters the active site and sits in between $\mathrm{H}_{2} \mathrm{O}_{2}$ and Pro139. At $10 \mathrm{~ns}$, the peroxide molecule swaps position with the water molecule: $\mathrm{H}_{2} \mathrm{O}_{2}$ moves to the vestibule of the active site, losing the aforementioned contact with the oxoferryl moiety and recovering the initial direct hydrogen bond with Pro139, whereas the water molecule sits in between $\mathrm{H}_{2} \mathrm{O}_{2}$, the oxoferryl and the distal histidine (configuration 3 ). Out of the three configurations explored, only configuration $\mathbf{2}$ is expected to be reactive, since the hydrogen peroxide molecule interacts with the oxoferryl unit as in catalase (Figure 3). Therefore, a snapshot of the classical MD with this configuration was chosen to start the subsequent QM/ MM MD simulations.

3.2. The Catalase-like Reaction Mechanism in HRP. The mechanism of $\mathrm{Cpd}$ I reduction by $\mathrm{H}_{2} \mathrm{O}_{2}$ in $\mathrm{HRP}$ was investigated with $\mathrm{QM}(\mathrm{DFT}) / \mathrm{MM}$ metadynamics using two collective variables (see Methods). To analyze the results obtained, it is useful to recall the meaning of the two collective variables used in the metadynamics simulation. CV1 (named oxygen formation) involves the distances between the two oxygen atoms of $\mathrm{H}_{2} \mathrm{O}_{2}$ and its two hydrogen atoms, thus it gives an idea of the degree formation of the product oxygen molecule. This variable ranges from a value of $\sim 2$ at the reactants state $(\mathbf{R})$, where each hydrogen atom is bound to its corresponding peroxide oxygen atom, to $\approx 0$ in the products state $(\mathbf{P})$, in which none of the hydrogen atoms is bound to the peroxide oxygens. The second collective variable, CV2 (named water formation) involves the distances between the oxoferryl oxygen atom and the two hydrogen atoms of $\mathrm{H}_{2} \mathrm{O}_{2}$, as well as the active site water molecule (wat). Thus, this variable, which changes from 0 to $\sim 2$ upon going from reactants to products, accounts for the formation of the second product of the catalatic reaction, a new water molecule.

The free energy landscape (FEL) reconstructed from the metadynamics simulation is shown in Figure 5a. The reactants $\left(\mathbf{R}\right.$, heme $\left.{ }^{+}-\mathrm{Fe}^{\mathrm{IV}}=\mathrm{O}+\mathrm{H}_{2} \mathrm{O}_{2}\right)$ and products $\left(\mathbf{P}\right.$, heme-Fe ${ }^{\mathrm{III}}+$ $\mathrm{H}_{2} \mathrm{O}+\mathrm{O}_{2}$ ) correspond to the two minima located on the bottom-right and upper-left-hand sides, respectively. Two minimum energy pathways can be drawn on the FEL: one that connects the reactants state with the products well (a reactive pathway) and another one that connects the reactants with the local minimum A2 (a dead-end pathway that does not reach the products). Representative configurations along each pathway are shown in Figures 6 and 7.

As shown in Figure 5a, the system can easily escape the reactants well via the lowest energy transition state $\mathbf{A l}\left(\Delta G^{\ddagger}=5\right.$

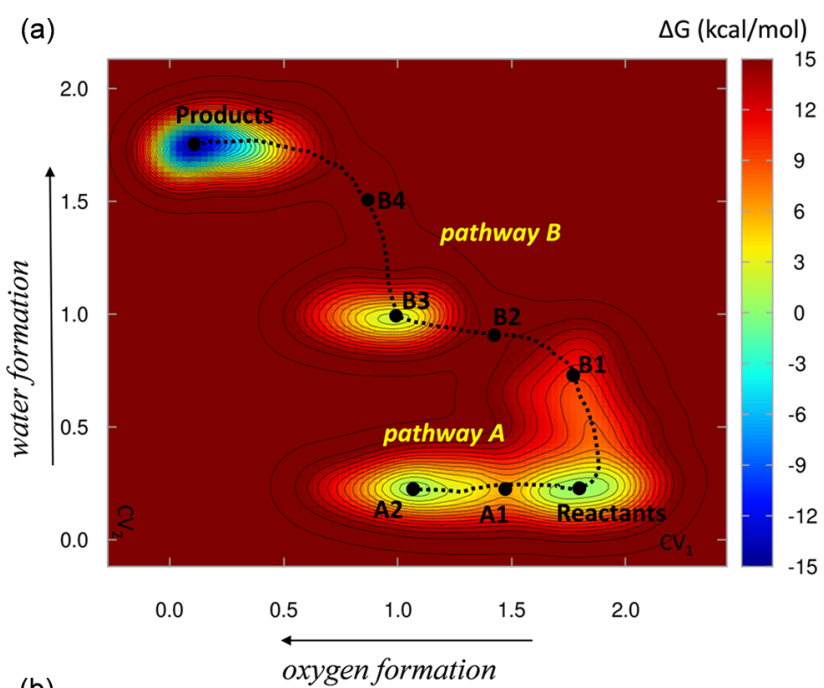

(b)

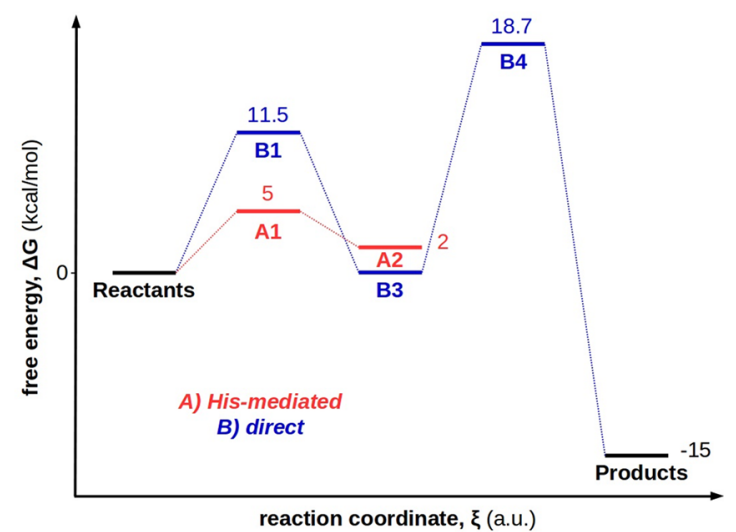

Figure 5. (a) Free energy landscape (FEL) of the catalase-like reaction in HRP reconstructed from the QM/MM metadynamics simulations. Contour lines are plotted at $1.2 \mathrm{kcal} / \mathrm{mol}$. (b) Schematic onedimensional graph including only the stationary points of the FEL and their relative energies.

$\mathrm{kcal} / \mathrm{mol}$ ) to reach the $\mathbf{A} \mathbf{2}$ minimum. This pathway, depicted in Figure 6, corresponds to the transfer of a peroxide proton to the distal histidine (note CV2 does not change from $\mathbf{R}$ to $\mathbf{A} 2$, as the bonding pattern of the oxoferryl oxygen remains unaltered). Species A2 is characterized by the shrinking of the $\mathrm{O}-\mathrm{O}$ distance (1.37 $\AA$ ) compared to the one of the $\mathrm{H}_{2} \mathrm{O}_{2}$ molecule at $\mathbf{R}$ ( 1.50 $\AA$ ), together with the presence of one unpaired electron in a $\pi$ orbital shared between the two peroxide oxygen atoms (see Table 1 and Table S1). The porphyrin radical, characteristic of Cpd I, disappears at A2. Therefore, formation of $\mathbf{A} 2$ consists of a proton transfer from $\mathrm{H}_{2} \mathrm{O}_{2}$ to $\mathrm{His} 42$ with a concomitant electron transfer from the peroxide to the porphyrin, forming a peroxyl radical $\left(\mathrm{HOO}^{\circ}\right)$.

To continue the catalase-like reaction, it is necessary that the histidine proton "travels" to the oxoferryl oxygen. However, the FEL does not show any direct pathway from $\mathbf{A} 2$ to the products. The direct transformation from A2 to $\mathbf{B} 3$ would present a free energy barrier much higher than the highest one sampled in our simulation, and thus the backward reaction to the reactants is the only favorable route to escape from A2. This is not surprising as the distance between the side chain of the distal His and the oxoferryl oxygen atom $(4.8 \AA)$ is too large for a facile proton transfer and the distal water molecule is not properly located to mediate such transfer. Indeed, a recent spectroscopic study ${ }^{40}$ has 

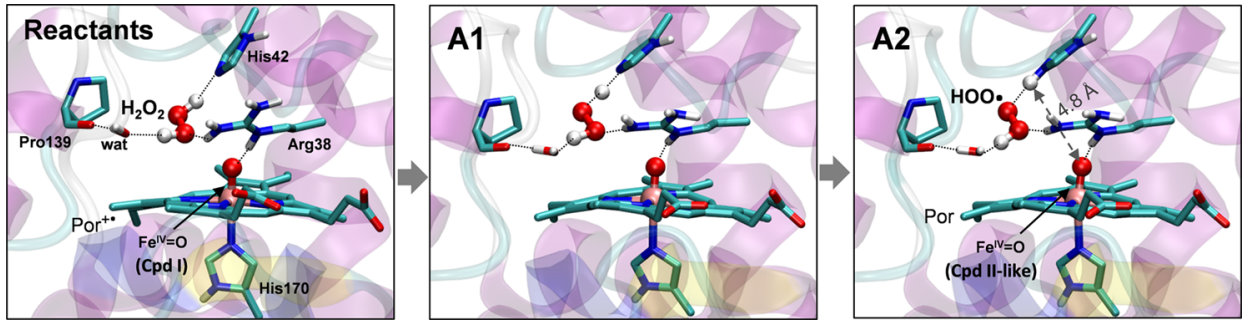

Figure 6. Representative snapshots along pathway A or the His-mediated pathway. Hydrogen atoms are omitted for clarity, except those involved in relevant hydrogen bonds.
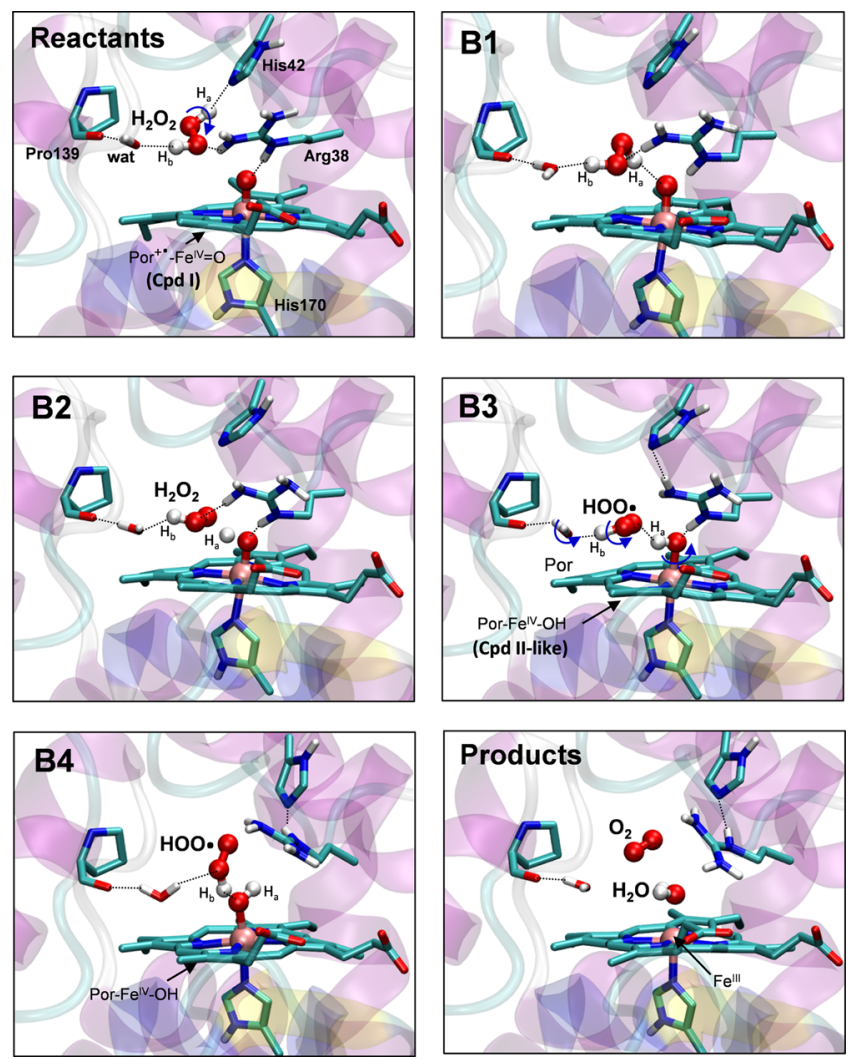

Figure 7. Representative snapshots along pathway $\mathbf{B}$ or the direct pathway. Hydrogen atoms are omitted for clarity, except those involved in relevant hydrogen bonds.

suggested that binding of reducing substrates to HRP Cpd I displaces water molecules from the active site and, as a consequence, water is unlikely to participate in Cpd I reduction. Therefore, whereas the His-mediated pathway in catalase is a reactive one (Figure 3), ${ }^{14}$ it corresponds to a dead-end for HRP. It is tempting to suggest that the $\mathbf{A} \mathbf{2}$ nonproductive intermediate may release ${ }^{-} \mathrm{OOH}$ or $\mathrm{O}_{2}^{-}$into the solution, given that superoxide dismutase (SOD) and other superoxide scavengers have been found to affect the HRP-catalyzed production of oxygen. ${ }^{41}$ The resulting "bare" Cpd II may in turn react with a second molecule of hydrogen peroxide and form Cpd III. Indeed, the spectroscopic signal of Cpd III has been detected when HRP is incubated in the presence of excess $\mathrm{H}_{2} \mathrm{O}_{2} .{ }^{9,41}$ Nevertheless, kinetic data has shown that the mechanism involving superoxide release and Cpd III formation is 2 orders of magnitude slower than the catalase-like pathway, ${ }^{41}$ and thus it was not explored in this work.

In contrast, pathway $\mathbf{B}$ does lead to the products of the catalase-like reaction (Figure 5), following a mechanism that reminds the direct pathway found for monofunctional catalase (Figure 3). The reactive mechanism, shown in Figure 7, begins with rotation of the peroxide in the heme pocket, breaking the hydrogen bond with His42 $\left(\mathrm{H}_{a} \cdots \mathrm{N}_{\varepsilon}\right.$-His42) and orienting $\mathrm{H}_{\mathrm{a}}$ toward the oxoferryl oxygen (B1). This facilitates the transfer of $\mathrm{H}_{\mathrm{a}}(\mathbf{B} 1 \rightarrow \mathbf{B} 2 \rightarrow$ B3 $)$, with a moderate energy barrier of $11.5 \mathrm{kcal}$ $\mathrm{mol}^{-1}$. As a consequence, the $\mathrm{Fe}-\mathrm{O}$ distance lengthens (from 1.68 to $1.83 \AA$ ) and the peroxide $\mathrm{O}-\mathrm{O}$ distance decreases (from 1.50 to $1.38 \AA$, see Tables 1 and S2), consistent with the formation of a peroxyl radical $\left(\mathrm{HOO}^{\bullet}\right)$. Analysis of the spin density distributions (Figure S2) reveals that, while the $\mathrm{H}_{a}$ proton transfers to the oxoferryl oxygen, the corresponding electron transfers to the iron-porphyrin, quenching the porphyrin radical. Therefore, the first step of the catalase-like reaction in HRP is the formation of a peroxyl radical species and a hydroxoferryl Cpd II, i.e., a Cpd II-like species, as it was found in catalase (Figure 3)

The evolution of the system from $\mathbf{B} 3$ to the final products, $\mathbf{P}$ is the rate-determining step of the global process, with a free energy barrier of $18.7 \mathrm{kcal} \mathrm{mol}^{-1}$ (Figure 5b). This value is in good agreement with the kinetic rate constant measured for this enzyme when incubated with $\mathrm{H}_{2} \mathrm{O}_{2}$ as sole substrate $(k=1.78 \pm$ $\left.0.12 \mathrm{~s}^{-1}, 4 \mathrm{~b} \Delta G^{\ddagger}=17.1 \mathrm{kcal} \mathrm{mol}^{-1}\right){ }^{42}$ Our results also confirm the conclusions of a spectrophotometric study ${ }^{41 a}$ that Cpd II conversion back to the resting state is rate-limiting in oxygen production by HRP. The free energy barrier turns out to be 6.7 $\mathrm{kcal} / \mathrm{mol}$ higher than the one previously computed for catalase, ${ }^{14}$ consistent with the lower catalatic activity measured for $\mathrm{HRP}^{41 \mathrm{~b}}$ compared to catalase. ${ }^{43}$

As shown in Figure 7, the overall process B3 $\rightarrow$ B4 $\rightarrow \mathbf{P}$ consists essentially in the reorientation of the peroxyl radical and the pocket water inside the active site cavity until the optimal alignment between the hydroxoferryl oxygen and the peroxyl radical is reached at the transition state $\mathbf{B} 4$. This is in line with the results in catalase, which showed that $\mathrm{H}$-bonding reorganization is the main determinant of the reaction barrier. Afterward, the second hydrogen atom, $\mathrm{H}_{\mathrm{b}}$, is transferred from $\mathrm{HOO}^{\bullet}$ to the hydroxoferryl unit forming the products of the reaction, oyxgen and water. As consequence, the $\mathrm{Fe}-\mathrm{O}$ distance lengthens from 1.83 to $3.10 \AA$ and the $\mathrm{O}-\mathrm{O}$ distance shrinks from 1.38 to $1.25 \AA$ (see Table 1 and Table S2), consistent with the formation of molecular oxygen. The spin-density analysis (Figure S2) also confirms the formation of triplet $\mathrm{O}_{2}$ and $\mathrm{H}_{2} \mathrm{O}$, as well as the reduction from $\mathrm{Fe}(\mathrm{IV})$ to $\mathrm{Fe}(\mathrm{III})$, recovering the resting state of the enzyme.

The dynamical description of the chemical reaction provided by the metadynamics ${ }^{44}$ approach allows capturing the motion of the active site residues during catalysis. ${ }^{1 \mathrm{~b}}$ Interestingly, the side chain of the distal His swings backward and the Arg side chain rotates and moves up (compare states $\mathbf{R}$ and $\mathbf{P}$ in Figure 7), leaving enough space to facilitate the departure of the $\mathrm{O}_{2}$ 
molecule from the active site cavity. The motion of these residues is also important to guide $\mathrm{HOO}^{\bullet}$ reorientation in the active site (compare states B3 and B4 in Figure 7), as discussed in the next section. Hints of the conformational motions observed in the metadynamics simulations can be already inferred from the available X-ray structures (see Tables S4 and S5 and Figure S3). However, only atomistic simulations can unraveled the pathway connecting the stationary states trapped in the crystal. ${ }^{1 \mathrm{Ib}}$

3.3. Hydrogen Bond Interactions in the Active Site. To identify the factors responsible for the reaction energy barrier of the catalatic reaction in HRP, we focused on the rate-determining step (from B3 to products) and quantified the change in hydrogen bond interactions in the active site (Figure S1) on going from minimum $\mathbf{B} 3$ to the transition state $\mathbf{B} 4$, following the procedure described in the Methods section 2.4. From the interaction energy changes $\left(\Delta E^{\ddagger}\right)$ we can estimate the contribution of a given hydrogen bond to the reorientation of the peroxyl radical and hydroxoferryl unit in the active site. The sum of all interaction energy differences $\left(\Delta E_{\text {TOTAL }}^{\#}\right)$ is expected to be an estimate to the reaction potential energy barrier. For comparison, we performed the same analysis for the catalase active site. The results of the interaction energy analysis are shown in Figure 8 and collected in Table S3. It should be noted that the wet active site of HRP contains two molecules, $\mathrm{HOO}^{\bullet}$ and wat, and thus we considered the interactions of both molecules with their neighboring active site residues (see Methods). In contrast, the dry active site of catalase only contains the peroxyl radical.
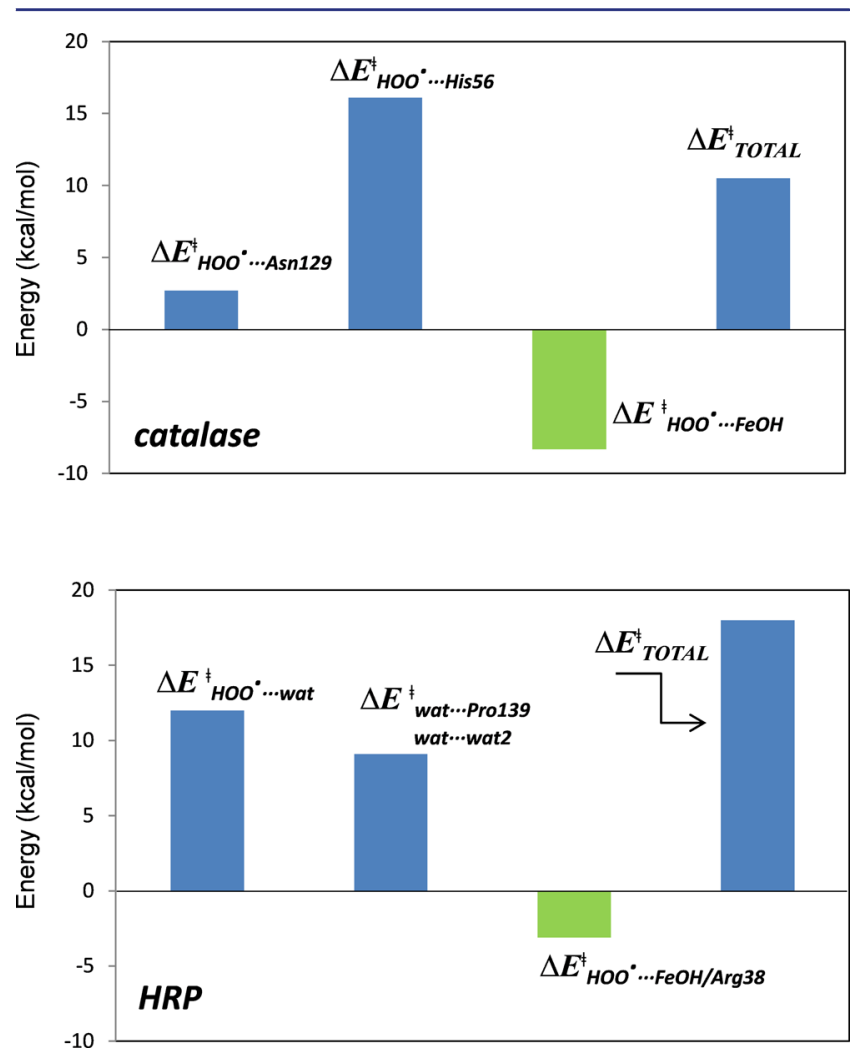

Figure 8. Interaction energy analysis between the peroxyl radical and the active site residues at the rate-limiting step of the reaction. The fragments considered are defined in Figure S1. Positive values of $\Delta E$ (blue color) indicate interactions that destabilize the transition state with respect to the corresponding minimum, while negative values (green color) denote stabilizing interactions.
In catalases, the main contribution to the reaction energy barrier turns out to be the weakening of the interactions of the peroxyl radical with the active site residues (mainly with His56) at the TS by $\sim 18 \mathrm{kcal} / \mathrm{mol}$ (Figure $8 \mathrm{a}$ ), which is only partially compensated by the strengthening of its interaction with the heme-FeOH group $\left(-8.3 \mathrm{kcal} \mathrm{mol}^{-1}\right)$. The picture differs in the case of HRP (Figure $8 \mathrm{~b}$ ), for which $\mathrm{HOO}^{\bullet}$ reorientation causes a more dramatic disruption of the hydrogen bond network than in catalase. In terms of interaction energies, the stabilizing interactions of both $\mathrm{HOO}^{\bullet}$ and the water molecule (wat) with their neighbors (Pro139 and another water molecule) decrease considerably (by $21.3 \mathrm{kcal} \mathrm{mol}^{-1}$ ) on going from the minimum (B3) to the TS (B4). On the other hand, the stabilizing interactions of the $\mathrm{HOO}^{\bullet}$ molecule (with the heme-FeOH$\cdots$ Arg38 fragment) increase only by $-3.1 \mathrm{kcal} \mathrm{mol}^{-1}$, and thus compensate only marginally the first term. Remarkably, summing up all contributions, the total destabilization of the TS $\left(\Delta E_{\text {TOTAL }}^{\#}\right.$ in Figure 8$)$ amounts to 10.5 and $18.2 \mathrm{kcal} \mathrm{mol}^{-1}$ for catalase and HRP, respectively, which is in good agreement with the values obtained for the free energy barriers using metadynamics $\left(12.0 \mathrm{kcal} \mathrm{mol}^{-1}\right.$ for catalase ${ }^{14}$ and $18.7 \mathrm{kcal} \mathrm{mol}^{-1}$ for HRP). This gives further validation to the fragment strategy employed here and, furthermore, supports the idea that the analysis of the distal site interactions captures the main factors that contribute to the different $\mathrm{Cpd}$ I reactivity of both enzymes.

In summary, we found that the reduction of $\mathrm{Cpd} \mathrm{I}$ by $\mathrm{H}_{2} \mathrm{O}_{2}$ in HRP consists of an initial hydrogen transfer to form a Cpd II-like species, followed by reorientation of the resulting peroxyl radical and the $\mathrm{FeOH}$ unit in the active site, with the subsequent transfer of the second hydrogen atom. This reaction pathway is similar to the "direct pathway" found for catalase (Figure 3), ${ }^{14}$ but the energy barrier of the reaction is significantly higher $(6.7 \mathrm{kcal} /$ $\mathrm{mol}$ ) for HRP due to a more hindered reorientation of the active site molecules. Furthermore, our results give support to the interpretation of the kinetic experiments in myoglobin $(\mathrm{Mb})$ mutants: ${ }^{43}$ the L29H/H64L Mb mutant, which mimics the HRP active site, was proposed to follow a direct pathway.

\section{SUMMARY AND CONCLUSIONS}

Using $\mathrm{QM}(\mathrm{DFT}) / \mathrm{MM}$ metadynamics simulations, we have uncovered the reaction mechanism for $\mathrm{Cpd}$ I reduction by $\mathrm{H}_{2} \mathrm{O}_{2}$ in HRP at atomic detail. The free energy landscape reconstructed from the metadynamics simulation, using two collective variables, shows two different minimum free energy pathways. Pathway A involves the distal His while B does not involve this residue. In this respect, the computed FEL is similar to the one obtained for catalase. ${ }^{14}$ However, whereas the His-mediated pathway or $\mathbf{A}$ in catalase is a reactive one, it corresponds to a dead-end for HRP, as the location of the distal His, being further away from the heme iron compared to catalases, precludes proton shuttling toward the oxoferryl unit. In contrast, the direct pathway or $\mathbf{B}$, involving a substantial reorientation of the peroxyl radical and the $\mathrm{FeOH}$ unit, leads to the products of the reaction in both HRP and catalases. Therefore, the global mechanism of Cpd I reduction by $\mathrm{H}_{2} \mathrm{O}_{2}$ in HRP consists in two consecutive hydrogen transfer steps, with a free energy barrier of $18.7 \mathrm{kcal}$ mol $^{-1}$, in very good agreement with experiments. ${ }^{41}$

The comparison of the reaction mechanism in HRP and catalase shows that the more crowded active site cavity in HRP, particularly designed to catalyze the oxidation of other substrates than $\mathrm{H}_{2} \mathrm{O}_{2}$, is the reason behind its lower catalatic activity. The presence of the positively charged distal Arg and a water molecule impedes the formation of interactions between $\mathrm{HOO}^{\bullet}$ 
and the active site residues strong enough to compensate the reorganization cost at the rate-determining step of the enzymatic reaction. In other words, the hydrogen bond network present in the "wet" active site cavity of horseradish peroxidase is disrupted more dramatically than in the "dry" catalase active site. This has a substantial impact on the height of the free energy barrier, which increases from $12 \mathrm{kcal} \mathrm{mol}^{-1}$ in catalase to $18.7 \mathrm{kcal} \mathrm{mol}^{-1}$ in HRP. Our results suggest that proper modification of the distal pocket, diminishing the number and/or the strength of hydrogen bond interactions (e.g., replacing the distal Arg by a less bulkier residue or a neutral one) could enhance the catalase-like activity of HRP and other heme proteins.

\section{ASSOCIATED CONTENT}

\section{S Supporting Information}

The Supporting Information is available free of charge on the ACS Publications website at DOI: 10.1021/jacs.5b06796.

Main structural parameters and spin density distributions of the stationary points of the free energy landscape. Details of the fragment analysis. Conformational analysis of the peroxidase crystallographic structures. (PDF)

\section{AUTHOR INFORMATION}

\section{Corresponding Authors}

*malfonsoprieto@gmail.com

*c.rovira@ub.edu

\section{Present Address}

"Université de Lorraine, Structure et Réactivité des Systèmes Moléculaires Complexes, Vandoeuvre-lés-Nancy, F-54506 France.

\section{Notes}

The authors declare no competing financial interest.

\section{ACKNOWLEDGMENTS}

This work was supported by grants CTQ2014-55174-P and 2014SGR-987 (to C.R.). M.A.-P. acknowledges the financial support from the Government of Catalonia (Commissioner for Universities and Research; Department of Innovation, Universities and Enterprise) and the European Union through a Beatriu de Pinós fellowship (BP-B 2013). We acknowledge the support from the COST Action CM1305 (ECOSTBio), as well as the computer support, technical expertise, and assistance provided by the Barcelona Supercomputing Center-Centro Nacional de Supercomputación (BSC-CNS). P.C. acknowledges the HPC-EUROPA2 project (project number 228398) with the support of the European Commission-Capacities Area-Research Infrastructures. The authors would also like to thank Dr. Pietro Vidossich for insightful discussions.

\section{REFERENCES}

(1) Zamocky, M.; Furtmuller, P. G.; Obinger, C. Antioxid. Redox Signaling 2008, 10, 1527-1548.

(2) Krainer, F. W.; Glieder, A. Appl. Microbiol. Biotechnol. 2015, 99, $1611-1625$.

(3) (a) Derat, E.; Cohen, S.; Shaik, S.; Altun, A.; Thiel, W. J. Am. Chem. Soc. 2005, 127, 13611-13621. (b) Dunford, H. B. Heme Peroxidases; John Wiley: New York, 1999.

(4) (a) Kim, S. H.; Perera, R.; Hager, L. P.; Dawson, J. H.; Hoffman, B. M. J. Am. Chem. Soc. 2006, 128, 5598-5599. (b) Ivancich, A.; Jouve, H. M.; Sartor, B.; Gaillard, J. Biochemistry 1997, 36, 9356-9364. (c) Chuang, W. J.; Vanwart, H. E. J. Biol. Chem. 1992, 267, 1329313301.
(5) (a) Derat, E.; Shaik, S. J. Am. Chem. Soc. 2006, 128, 8185-8198. (b) Dunford, H. B.; Dunford, H. B. Peroxidases and Catalases: Biochemistry, Biophysics, Biotechnology, and Physiology, 2nd ed.; Wiley: Hoboken, NJ, 2010.

(6) Nicholls, P.; Fita, I.; Loewen, P. C. In Advances in Inorganic Chemistry; Sykes, A. G. G. M., Ed.; Academic Press: Waltham, MA, 2001; pp 51-106.

(7) (a) Matsui, T.; Ozaki, S.; Liong, E.; Phillips, G. N., Jr.; Watanabe, Y. J. Biol. Chem. 1999, 274, 2838-2844. (b) Hernandez-Ruiz, J.; Arnao, M. B.; Hiner, A. N.; Garcia-Canovas, F.; Acosta, M. Biochem. J. 2001, 354, $107-114$.

(8) (a) Matsunaga, I.; Shiro, Y. Curr. Opin. Chem. Biol. 2004, 8, 127132. (b) Nastri, F.; Lista, L.; Ringhieri, P.; Vitale, R.; Faiella, M.; Andreozzi, C.; Travascio, P.; Maglio, O.; Lombardi, A.; Pavone, V. Chem. - Eur. J. 2011, 17, 4444-4453.

(9) Vlasits, J.; Jakopitsch, C.; Bernroitner, M.; Zamocky, M.; Furtmuller, P. G.; Obinger, C. Arch. Biochem. Biophys. 2010, 500, 74-81.

(10) (a) Dawson, J. H. Science 1988, 240, 433-439. (b) de Visser, S. P.; Shaik, S.; Sharma, P. K.; Kumar, D.; Thiel, W. J. Am. Chem. Soc. 2003, 125, 15779-15788. (c) Chelikani, P.; Fita, I.; Loewen, P. C. Cell. Mol. Life Sci. 2004, 61, 192-208.

(11) (a) Alfonso-Prieto, M.; Vidossich, P.; Rovira, C. Arch. Biochem. Biophys. 2012, 525, 121-130. (b) Vidossich, P.; Alfonso-Prieto, M.; Carpena, X.; Fita, I.; Loewen, P. C.; Rovira, C. Arch. Biochem. Biophys. 2010, 500, 37-44.

(12) (a) Takahashi, A.; Kurahashi, T.; Fujii, H. Inorg. Chem. 2009, 48, 2614-2625. (b) Robert, A.; Loock, B.; Momenteau, M.; Meunier, B. Inorg. Chem. 1991, 30, 706-711.

(13) (a) Silaghi-Dumitrescu, R. J. Porphyrins Phthalocyanines 2010, 14, 371-374. (b) Vidossich, P.; Alfonso-Prieto, M.; Rovira, C. J. Inorg. Biochem. 2012, 117, 292-297.

(14) Alfonso-Prieto, M.; Biarnés, X.; Vidossich, P.; Rovira, C. J. Am. Chem. Soc. 2009, 131, 11751-11761.

(15) Fita, I.; Rossmann, M. G. J. Mol. Biol. 1985, 185, 21-37.

(16) Loewen, P. C.; Carpena, X.; Vidossich, P.; Fita, I.; Rovira, C. J. Am. Chem. Soc. 2014, 136, 7249-7252.

(17) Berglund, G. I.; Carlsson, G. H.; Smith, A. T.; Szoke, H.; Henriksen, A.; Hajdu, J. Nature 2002, 417, 463-468.

(18) (a) Olsson, M. H. M.; Søndergaard, C. R.; Rostkowski, M.; Jensen, J. H. J. Chem. Theory Comput. 2011, 7, 525-537. (b) Søndergaard, C. R.; Olsson, M. H. M.; Rostkowski, M.; Jensen, J. H. J. Chem. Theory Comput. 2011, 7, 2284-2295.

(19) Derat, E.; Shaik, S. J. Am. Chem. Soc. 2006, 128, 13940-13949.

(20) Casadei, C. M.; Gumiero, A.; Metcalfe, C. L.; Murphy, E. J.; Basran, J.; Concilio, M. G.; Teixeira, S. C.; Schrader, T. E.; Fielding, A. J.; Ostermann, A.; Blakeley, M. P.; Raven, E. L.; Moody, P. C. Science 2014, 345, 193-197.

(21) Cornell, W. D.; Cieplak, P.; Bayly, C. I.; Gould, I. R.; Merz, K. M.; Ferguson, D. M.; Spellmeyer, D. C.; Fox, T.; Caldwell, J. W.; Kollman, P. A. J. Am. Chem. Soc. 1995, 117, 5179-5197.

(22) Jorgensen, W. L.; Chandrasekhar, J.; Madura, J. D.; Impey, R. W.; Klein, M. L. J. Chem. Phys. 1983, 79, 926-935.

(23) Banci, L.; Gori-Savellini, G.; Turano, P. Eur. J. Biochem. 1997, 249, $716-723$.

(24) Essmann, U.; Perera, L.; Berkowitz, M. L.; Darden, T.; Lee, H.; Pedersen, L. G. J. Chem. Phys. 1995, 103, 8577-8593.

(25) Kubo, R.; Hashitsume, N.; Toda, M. Statistical Physics II: Nonequilibrium Statistical Mechanics, 2nd ed.; Springer: Berlin, 1995.

(26) (a) Jones, P.; Dunford, H. B. J. Inorg. Biochem. 2005, 99, 22922298. (b) Adamczyk, K.; Simpson, N.; Greetham, G. M.; Gumiero, A.; Walsh, M. A.; Towrie, M.; Parker, A. W.; Hunt, N. T. Chem. Sci. 2015, 6, 505-516.

(27) Derat, E.; Shaik, S.; Rovira, C.; Vidossich, P.; Alfonso-Prieto, M. J. Am. Chem. Soc. 2007, 129, 6346-6347.

(28) Phillips, J. C.; Braun, R.; Wang, W.; Gumbart, J.; Tajkhorshid, E.; Villa, E.; Chipot, C.; Skeel, R. D.; Kale, L.; Schulten, K. J. Comput. Chem. 2005, 26, 1781-1802.

(29) Humphrey, W.; Dalke, A.; Schulten, K. J. Mol. Graphics 1996, 14, 33-38. 
(30) VandeVondele, J.; Krack, M.; Mohamed, F.; Parrinello, M.; Chassaing, T.; Hutter, J. Comput. Phys. Commun. 2005, 167, 103-128.

(31) (a) Laino, T.; Mohamed, F.; Laio, A.; Parrinello, M. J. Chem. Theory Comput. 2005, 1, 1176-1184. (b) Laio, A.; VandeVondele, J.; Rothlisberger, U. J. Chem. Phys. 2002, 116, 6941-6947.

(32) VandeVondele, J.; Hutter, J. J. Chem. Phys. 2007, 127, 114105.

(33) (a) Goedecker, S.; Teter, M.; Hutter, J. Phys. Rev. B: Condens. Matter Mater. Phys. 1996, 54, 1703-1710. (b) Hartwigsen, C.; Goedecker, S.; Hutter, J. Phys. Rev. B: Condens. Matter Mater. Phys. 1998, 58, 3641-3662.

(34) (a) Becke, A. D. Phys. Rev. A: At., Mol., Opt. Phys. 1988, 38, 30983100. (b) Lee, C. T.; Yang, W. T.; Parr, R. G. Phys. Rev. B: Condens. Matter Mater. Phys. 1988, 37, 785-789.

(35) Benecky, M. J.; Frew, J. E.; Scowen, N.; Jones, P.; Hoffman, B. M. Biochemistry 1993, 32, 11929-11933.

(36) Bussi, G.; Donadio, D.; Parrinello, M. J. Chem. Phys. 2007, 126, 014101.

(37) (a) Laio, A.; Parrinello, M. Proc. Natl. Acad. Sci. U. S. A. 2002, 99, 12562-12566. (b) Iannuzzi, M.; Laio, A.; Parrinello, M. Phys. Rev. Lett. 2003, 90, 238302.

(38) (a) Laio, A.; Rodriguez-Fortea, A.; Gervasio, F. L.; Ceccarelli, M.; Parrinello, M. J. Phys. Chem. B 2005, 109, 6714-6721. (b) Nair, N. N.; Schreiner, E.; Marx, D. J. Am. Chem. Soc. 2008, 130, 14148-14160.

(39) (a) Morokuma, K.; Kitaura, K. Int. J. Quantum Chem. 1976, 10, 325-340. (b) Ziegler, T.; Rauk, A. Theor. Chim. Acta. 1977, 46, 1-10. (c) Ess, D. H.; Houk, K. N. J. Am. Chem. Soc. 2007, 129, 10646-10647. (d) Usharani, D.; Lacy, D. C.; Borovik, A. S.; Shaik, S. J. Am. Chem. Soc. 2013, 135, 17090-17104. (e) Fernandez, I.; Bickelhaupt, F. M. Chem. Soc. Rev. 2014, 43, 4953-4967.

(40) Simpson, N.; Adamczyk, K.; Hithell, G.; Shaw, D. J.; Greetham, G. M.; Towrie, M.; Parker, A. W.; Hunt, N. T. Faraday Discuss. 2015, 177, 163-179.

(41) (a) Baker, C. J.; Deahl, K.; Domek, J.; Orlandi, E. W. Arch. Biochem. Biophys. 2000, 382, 232-237. (b) Hiner, A. N.; HernandezRuiz, J.; Williams, G. A.; Arnao, M. B.; Garcia-Canovas, F.; Acosta, M. Arch. Biochem. Biophys. 2001, 392, 295-302.

(42) According to conventional TST: $k=\left(k_{\mathrm{B}} T / h\right) \exp \left(-\Delta G^{\ddagger} / R T\right)$, where $\Delta G^{\ddagger}$ is the free energy barrier.

(43) Kato, S.; Ueno, T.; Fukuzumi, S.; Watanabe, Y. J. Biol. Chem. 2004, 279, 52376-52381.

(44) Barducci, A.; Bonomi, M.; Parrinello, M. WIREs Comput. Mol. Sci. 2011, 1, 826-843.

(45) Bader, R. F. W. Atoms in Molecules: A Quantum Theory, corrected paperback ed.; Clarendon Press: Oxford, 1994.

(46) Alfonso-Prieto, M.; Borovik, A.; Carpena, X.; Murshudov, G.; Melik-Adamyan, W.; Fita, I.; Rovira, C.; Loewen, P. C. J. Am. Chem. Soc. 2007, 129, 4193-4205. 